\title{
Further refinements of reversed AM-GM operator inequalities
}

\author{
Yonghui Ren ${ }^{1 *}$ and Pengtong $\mathrm{Li}^{1}$
}

"Correspondence: 2294719246@qq.com

'Department of Mathematics, Nanjing University of Aeronautics and Astronautics, Nanjing, China

\section{Springer}

\begin{abstract}
In this paper, we shall give further improvements of reversed AM-GM operator inequalities due to Yang et al. (Math. Slovaca 69:919-930, 2019) for matrices and positive linear map.
\end{abstract}

MSC: 47A63; 47A60

Keywords: Positive linear map; Hilbert-Schmidt norms; Determinant

\section{Introduction}

Throughout this paper, let $m, M$ be scalars and $I$ be the identity operator. Other capital letters are used to denote the general elements of the $C^{*}$ algebra $B(\mathcal{H})$ of all bounded linear operators acting on a Hilbert space $(\mathcal{H},\langle\cdot, \cdot\rangle)$. Also $A \geq 0$ means that the operator $A$ is positive. We say that $A \geq B(A \leq B)$ if $A-B \geq 0(A-B \leq 0)$. A linear map $\Phi: B(\mathcal{H}) \rightarrow B(\mathcal{H})$ is called positive (strictly positive) if $\Phi(A) \geq 0(\Phi(A)>0)$ whenever $A \geq 0(A>0)$, and $\Phi$ is said to be unital if $\Phi(I)=I$.

If $A, B \in B(\mathcal{H})$ are two positive operators, then the operator weighted arithmetic and geometric means are defined as $A \nabla_{v} B=(1-v) A+v B$ and $A \sharp_{v} B=A^{\frac{1}{2}}\left(A^{-\frac{1}{2}} B A^{-\frac{1}{2}}\right)^{v} A^{\frac{1}{2}}$ for $v \in[0,1]$, respectively, denoted by $A \nabla B$ and $A \sharp B$ for brevity when $v=\frac{1}{2}$. The Kantorovich constant is defined by $K(t, 2)=\frac{(t+1)^{2}}{4 t}$ for $t>0$. What's more, the relative operator entropy of $A$ and $B$ is defined as $S(A \mid B)=A^{\frac{1}{2}} \log \left(A^{-\frac{1}{2}} B A^{-\frac{1}{2}}\right) A^{\frac{1}{2}}$. For $A=\left(a_{i j}\right) \in M_{n}$, the HilbertSchmidt norm is defined by $\|A\|_{2}=\sqrt{\sum_{i, j=1}^{n} a_{i j}^{2}}$. As we all know that $\|\cdot\|_{2}$ has the unitary invariance property: $\|U A V\|_{2}=\|A\|_{2}$ for all $A \in M_{n}$ and unitary matrices $U, V \in M_{n}$. The singular values of a matrix $A$ is defined by $s_{j}(A), j=1,2, \ldots, n$, and arranged in a nonincreasing order.

It is well known that the AM-GM inequality reads

$$
\frac{A+B}{2} \geq A \sharp B
$$

for any two positive operators $A, B$.

Lin [10] gave a reversed AM-GM inequality involving unital positive linear maps

$$
\Phi\left(\frac{A+B}{2}\right) \leq K(h, 2) \Phi(A \sharp B)
$$

(c) The Author(s) 2020. This article is licensed under a Creative Commons Attribution 4.0 International License, which permits use sharing, adaptation, distribution and reproduction in any medium or format, as long as you give appropriate credit to the original author(s) and the source, provide a link to the Creative Commons licence, and indicate if changes were made. The images or other third party material in this article are included in the article's Creative Commons licence, unless indicated otherwise in a credit line to the material. If material is not included in the article's Creative Commons licence and your intended use is not permitted by statutory regulation or exceeds the permitted use, you will need to obtain permission directly from the copyright holder. To view a copy of this licence, visit http://creativecommons.org/licenses/by/4.0/. 
for $0<m I \leq A, B \leq M I$ and $K(h, 2)=\frac{(h+1)^{2}}{4 h}$ with $h=\frac{M}{m}$.

As we all know, for any two positive operators $A$ and $B$,

$$
A \geq B \quad \nRightarrow \quad A^{p} \geq B^{p}
$$

for $p>1$. To our surprise, Lin [10] showed that a reversed version of the operator AM-GM inequality can be squared: for $0<m I \leq A, B \leq M I$,

$$
\Phi^{2}\left(\frac{A+B}{2}\right) \leq\left(\frac{(M+m)^{2}}{4 M m}\right)^{2} \Phi^{2}(A \sharp B)
$$

and

$$
\Phi^{2}\left(\frac{A+B}{2}\right) \leq\left(\frac{(M+m)^{2}}{4 M m}\right)^{2}(\Phi(A) \sharp \Phi(B))^{2},
$$

where $\Phi$ is a unital positive linear map. So we can easily get the following inequalities by Lemma 2.8 ( $\mathrm{L}-\mathrm{H}$ inequality):

$$
\Phi^{p}\left(\frac{A+B}{2}\right) \leq\left(\frac{(M+m)^{2}}{4 M m}\right)^{p} \Phi^{p}(A \sharp B)
$$

and

$$
\Phi^{p}\left(\frac{A+B}{2}\right) \leq\left(\frac{(M+m)^{2}}{4 M m}\right)^{p}(\Phi(A) \sharp \Phi(B))^{p}
$$

for $0 \leq p \leq 2$. Fu and He [5] generalized (1.6) and (1.7) for $p \geq 2$,

$$
\Phi^{p}\left(\frac{A+B}{2}\right) \leq\left(\frac{(M+m)^{2}}{4^{\frac{2}{p}} M m}\right)^{p} \Phi^{p}(A \sharp B)
$$

and

$$
\Phi^{p}\left(\frac{A+B}{2}\right) \leq\left(\frac{(M+m)^{2}}{4^{\frac{2}{p}} M m}\right)^{p}(\Phi(A) \sharp \Phi(B))^{p},
$$

where $0<m I \leq A, B \leq M I$. Bakherad [2] further improved (1.6)-(1.9) as follows:

$$
\Phi^{p}\left(A \nabla_{\nu} B+2 r M m\left(A^{-1} \nabla B^{-1}-A^{-1} \sharp B^{-1}\right)\right) \leq \alpha^{p} \Phi^{p}\left(A \sharp_{\nu} B\right)
$$

and

$$
\Phi^{p}\left(A \nabla_{\nu} B+2 r M m\left(A^{-1} \nabla B^{-1}-A^{-1} \sharp B^{-1}\right)\right) \leq \alpha^{p}\left(\Phi(A) \sharp_{\nu} \Phi(B)\right)^{p}
$$

for $0<m I \leq A, B \leq M I, p \geq 0, \alpha=\max \left\{\frac{(M+m)^{2}}{4 M m}, \frac{(M+m)^{2}}{4^{\frac{2}{p}} M m}\right\}, v \in[0,1], r=\min \{v, 1-v\}$ and $\Phi$ being a unital positive linear map.

Recently, Yang et al. [12] gave some further refinements to the above:

$$
\Phi^{p}\left(A \nabla_{\nu} B+M m\left(G\left(A^{-1} \sharp_{\nu} B^{-1}\right) G^{*}+2 r\left(A^{-1} \nabla B^{-1}-A^{-1} \sharp B^{-1}\right)\right)\right) \leq \alpha^{p} \Phi^{p}\left(A \sharp_{\nu} B\right)
$$


and

$$
\begin{aligned}
& \Phi^{p}\left(A \nabla_{\nu} B+M m\left(G\left(A^{-1} \sharp_{\nu} B^{-1}\right) G^{*}+2 r\left(A^{-1} \nabla B^{-1}-A^{-1} \sharp B^{-1}\right)\right)\right) \\
& \quad \leq \alpha^{p}\left(\Phi(A) \sharp_{\nu} \Phi(B)\right)^{p},
\end{aligned}
$$

where $0<m I \leq A, B \leq M I, \Phi$ is a positive unital linear map on $B(\mathcal{H}), v \in[0,1]$ and $p>$ $0, r=\min \{v, 1-v\}, \alpha=\max \left\{\frac{(M+m)^{2}}{4 M m}, \frac{(M+m)^{2}}{4^{\frac{2}{p}} M m}\right\}, G=\frac{\sqrt{L(2 v)}}{2} A^{-1} S(A \mid B), L(t)$ is 1-periodic, and $L(t)=\frac{t^{2}}{2}\left(\frac{1-t}{t}\right)^{2 t}$ for $t \in[0,1]$. In fact, we can get (1.10) from (1.12) and (1.11) from (1.13) when $v=\frac{1}{2}$, respectively. For more information about AM-GM operator inequalities, we refer the readers to $[9,11,13-18]$ and the references therein.

In this paper, we shall give further improvements of (1.12) and (1.13) for positive linear maps. We also give some inequalities for Hilbert-Schmidt norms and determinants.

\section{Main results}

Firstly, we give some further refinements of the corresponding results in [12] for scalars and Hilbert-Schmidt norms. Before that, we state a lemma.

Lemma 2.1 ([7]) Let $a, b>0$. Then

$$
\left(1+L(v)(\log a-\log b)^{2}\right) a^{1-v} b^{v} \leq(1-v) a+v b,
$$

where

$$
L(v)= \begin{cases}\frac{v^{2}}{2}\left(\frac{1-v}{v}\right)^{2 v}, & 0<v<1, \\ 0, & v=0,1 .\end{cases}
$$

Theorem 2.2 Let $a, b>0, v \in[0,1], Q(v)=\frac{L(8 v)}{64}\left(\log \frac{b}{a}\right)^{2}$. We have:

(1) If $r=\min \{4 v, 1-4 v\}$ and $0 \leq v \leq \frac{1}{4}$, then

$$
\begin{aligned}
(1 & +Q(v)) a^{1-v} b^{v}+v(\sqrt{a}-\sqrt{b})^{2}+2 v(\sqrt{a}-\sqrt[4]{a b})^{2}+r\left(\sqrt{a}-\sqrt[8]{a^{3} b}\right)^{2} \\
& \leq(1-v) a+v b
\end{aligned}
$$

(2) If $r=\min \{2-4 v, 4 v-1\}$ and $\frac{1}{4} \leq v \leq \frac{1}{2}$, then

$$
\begin{aligned}
(1 & +Q(v)) a^{1-v} b^{v}+v(\sqrt{a}-\sqrt{b})^{2}+(1-2 v)(\sqrt{a}-\sqrt[4]{a b})^{2}+r\left(\sqrt[4]{a b}-\sqrt[8]{a^{3} b}\right)^{2} \\
& \leq(1-v) a+v b
\end{aligned}
$$

(3) If $r=\min \{3-4 v, 4 v-2\}$ and $\frac{1}{2} \leq v \leq \frac{3}{4}$, then

$$
\begin{aligned}
(1 & +Q(v)) a^{1-v} b^{v}+(1-v)(\sqrt{a}-\sqrt{b})^{2}+(2 v-1)(\sqrt{b}-\sqrt[4]{a b})^{2}+r\left(\sqrt[4]{a b}-\sqrt[8]{a b^{3}}\right)^{2} \\
& \leq(1-v) a+v b
\end{aligned}
$$

(4) If $r=\min \{4-4 v, 4 v-3\}$ and $\frac{3}{4} \leq v \leq 1$, then

$$
\begin{aligned}
(1+ & Q(v)) a^{1-v} b^{v}+(1-v)(\sqrt{a}-\sqrt{b})^{2}+(2-2 v)(\sqrt{b}-\sqrt[4]{a b})^{2}+r\left(\sqrt{b}-\sqrt[8]{a b^{3}}\right)^{2} \\
& \leq(1-v) a+v b .
\end{aligned}
$$


Proof (1) When $0 \leq v \leq \frac{1}{8}$, then $r=4 v$, and

$$
\begin{aligned}
(1 & -v) a+v b-v(\sqrt{a}-\sqrt{b})^{2}-2 v(\sqrt{a}-\sqrt[4]{a b})^{2}-r\left(\sqrt{a}-\sqrt[8]{a^{3} b}\right)^{2} \\
& =(1-8 v) a+8 v a^{\frac{7}{8}} b^{\frac{1}{8}} \\
& \geq\left(1+\frac{L(8 v)}{64}\left(\log \frac{b}{a}\right)^{2}\right) a^{1-v} b^{v} \quad(\text { by }(2.1)) .
\end{aligned}
$$

When $\frac{1}{8} \leq v \leq \frac{1}{4}$, then $r=1-4 v$, and

$$
\begin{aligned}
(1 & -v) a+v b-v(\sqrt{a}-\sqrt{b})^{2}-2 v(\sqrt{a}-\sqrt[4]{a b})^{2}-r\left(\sqrt{a}-\sqrt[8]{a^{3} b}\right)^{2} \\
& =(2-8 v) a^{\frac{7}{8}} b^{\frac{1}{8}}+(8 v-1) a^{\frac{3}{4}} b^{\frac{1}{4}} \\
& \geq\left(1+\frac{L(8 v-1)}{64}\left(\log \frac{b}{a}\right)^{2}\right) a^{1-v} b^{v} \\
& =\left(1+\frac{L(8 v)}{64}\left(\log \frac{b}{a}\right)^{2}\right) a^{1-v} b^{v},
\end{aligned}
$$

where the last equality from $L(v)$ is a 1 -periodic function and $L(v)=L(1-v)$ for $v \in[0,1]$.

(2) When $\frac{1}{4} \leq v \leq \frac{3}{8}$, then $r=4 v-1$, and

$$
\begin{aligned}
(1 & -v) a+v b-v(\sqrt{a}-\sqrt{b})^{2}-(1-2 v)(\sqrt{a}-\sqrt[4]{a b})^{2}-r\left(\sqrt[4]{a b}-\sqrt[8]{a^{3} b}\right)^{2} \\
& =(3-8 v) a^{\frac{3}{4}} b^{\frac{1}{4}}+(8 v-2) a^{\frac{5}{8}} b^{\frac{3}{8}} \\
& \geq\left(1+\frac{L(8 v-2)}{64}\left(\log \frac{b}{a}\right)^{2}\right) a^{1-v} b^{v} \\
& =\left(1+\frac{L(8 v)}{64}\left(\log \frac{b}{a}\right)^{2}\right) a^{1-v} b^{v}
\end{aligned}
$$

When $\frac{3}{8} \leq v \leq \frac{1}{2}$, then $r=2-4 v$, and

$$
\begin{aligned}
(1 & -v) a+v b-v(\sqrt{a}-\sqrt{b})^{2}-(1-2 v)(\sqrt{a}-\sqrt[4]{a b})^{2}-r\left(\sqrt[4]{a b}-\sqrt[8]{a^{3} b}\right)^{2} \\
& =(4-8 v) a^{\frac{5}{8}} b^{\frac{3}{8}} a^{\frac{1}{2}} b^{\frac{1}{2}}+(8 v-3) a^{\frac{1}{2}} b^{\frac{1}{2}} \\
& \geq\left(1+\frac{L(8 v-3)}{64}\left(\log \frac{b}{a}\right)^{2}\right) a^{1-v} b^{v} \\
& =\left(1+\frac{L(8 v)}{64}\left(\log \frac{b}{a}\right)^{2}\right) a^{1-v} b^{v}
\end{aligned}
$$

Here, we completed the proof of Theorem 2.2 when $0 \leq v \leq \frac{1}{2}$. Substituting $a$ by $b$ and $v$ by $1-v$ in (2.2) and (2.3), we can get (2.4) and (2.5) easily, so we omit the details.

The following corollary is a direct consequence of Theorem 2.2 by substituting $a$ by $a^{2}$ and $b$ by $b^{2}$.

Corollary 2.3 Let $a, b>0, v \in[0,1], F(v)=\frac{L(8 v)}{16}\left(\log \frac{b}{a}\right)^{2}$. We have: 
(1) If $r=\min \{4 v, 1-4 v\}$ and $0 \leq v \leq \frac{1}{4}$, then

$$
\begin{aligned}
(1+ & +F(v))\left(a^{1-v} b^{v}\right)^{2}+v^{2}(a-b)^{2}+2 v(a-\sqrt{a b})^{2}+r\left(a-\sqrt[4]{a^{3} b}\right)^{2} \\
& \leq((1-v) a+v b)^{2}
\end{aligned}
$$

(2) If $r=\min \{2-4 v, 4 v-1\}$ and $\frac{1}{4} \leq v \leq \frac{1}{2}$, then

$$
\begin{aligned}
(1 & +F(v))\left(a^{1-v} b^{v}\right)^{2}+v^{2}(a-b)^{2}+(1-2 v)(a-\sqrt{a b})^{2}+r\left(\sqrt{a b}-\sqrt[4]{a^{3} b}\right)^{2} \\
& \leq((1-v) a+v b)^{2}
\end{aligned}
$$

(3) If $r=\min \{3-4 v, 4 v-2\}$ and $\frac{1}{2} \leq v \leq \frac{3}{4}$, then

$$
\begin{aligned}
(1 & +F(v))\left(a^{1-v} b^{v}\right)^{2}+(1-v)^{2}(a-b)^{2}+(2 v-1)(b-\sqrt{a b})^{2}+r\left(\sqrt{a b}-\sqrt[4]{a b^{3}}\right)^{2} \\
& \leq((1-v) a+v b)^{2}
\end{aligned}
$$

(4) If $r=\min \{4-4 v, 4 v-3\}$ and $\frac{3}{4} \leq v \leq 1$, then

$$
\begin{aligned}
(1+ & +F(v))\left(a^{1-v} b^{v}\right)^{2}+(1-v)^{2}(a-b)^{2}+(2-2 v)(b-\sqrt{a b})^{2}+r\left(b-\sqrt[4]{a b^{3}}\right)^{2} \\
& \leq((1-v) a+v b)^{2} .
\end{aligned}
$$

Theorem 2.4 Let $A, B, X \in M_{n}$ be such that $0<m I \leq A \leq m^{\prime} I<M^{\prime} I \leq B \leq M I, h=\frac{M^{\prime}}{m^{\prime}}$, $W(v)=\frac{L(8 v)}{16}(\log h)^{2}$. We have:

(1) If $r=\min \{4 v, 1-4 v\}$ and $0 \leq v \leq \frac{1}{4}$, then

$$
\begin{aligned}
& \|(1-v) A X+v X B\|_{2}^{2} \\
& \geq v^{2}\|A X-X B\|_{2}^{2}+2 v\left\|A X-A^{\frac{1}{2}} X B^{\frac{1}{2}}\right\|_{2}^{2} \\
& \quad+r\left\|A X-A^{\frac{3}{4}} X B^{\frac{1}{4}}\right\|_{2}^{2}+(1+W(v))\left\|A^{1-v} X B^{v}\right\|_{2}^{2} ;
\end{aligned}
$$

(2) If $r=\min \{2-4 v, 4 v-1\}$ and $\frac{1}{4} \leq v \leq \frac{1}{2}$, then

$$
\begin{aligned}
\|(1-v) A X+v X B\|_{2}^{2} & \\
\geq & v^{2}\|A X-X B\|_{2}^{2}+(1-2 v)\left\|A X-A^{\frac{1}{2}} X B^{\frac{1}{2}}\right\|_{2}^{2} \\
& +r\left\|A^{\frac{1}{2}} X B^{\frac{1}{2}}-A^{\frac{3}{4}} X B^{\frac{1}{4}}\right\|_{2}^{2}+(1+W(v))\left\|A^{1-v} X B^{v}\right\|_{2}^{2} ;
\end{aligned}
$$

(3) If $r=\min \{3-4 v, 4 v-2\}$ and $\frac{1}{2} \leq v \leq \frac{3}{4}$, then

$$
\begin{aligned}
& \|(1-v) A X+v X B\|_{2}^{2} \\
& \geq(1-v)^{2}\|A X-X B\|_{2}^{2}+(2 v-1)\left\|X B-A^{\frac{1}{2}} X B^{\frac{1}{2}}\right\|_{2}^{2} \\
& \quad+r\left\|A^{\frac{1}{2}} X B^{\frac{1}{2}}-A^{\frac{1}{4}} X B^{\frac{3}{4}}\right\|_{2}^{2}+(1+W(v))\left\|A^{1-v} X B^{v}\right\|_{2}^{2} ;
\end{aligned}
$$


(4) If $r=\min \{4-4 v, 4 v-3\}$ and $\frac{3}{4} \leq v \leq 1$, then

$$
\begin{aligned}
& \|(1-v) A X+v X B\|_{2}^{2} \\
& \geq(1-v)^{2}\|A X-X B\|_{2}^{2}+(2-2 v)\left\|X B-A^{\frac{1}{2}} X B^{\frac{1}{2}}\right\|_{2}^{2} \\
& \quad+r\left\|X B-A^{\frac{1}{4}} X B^{\frac{3}{4}}\right\|_{2}^{2}+(1+W(v))\left\|A^{1-v} X B^{v}\right\|_{2}^{2}
\end{aligned}
$$

Proof Since $A$ and $B$ are strictly positive-definite matrices, it follows by the spectral decomposition theorem that there exist unitary matrices $U, V \in M_{n}$, such that $A=U \Lambda_{1} U^{*}$, $B=V \Lambda_{2} V^{*}$, where $\Lambda_{1}=\operatorname{diag}\left(\lambda_{1}, \lambda_{2}, \ldots, \lambda_{n}\right), \Lambda_{2}=\operatorname{diag}\left(\eta_{1}, \eta_{2}, \ldots, \eta_{n}\right)$ with $\lambda_{i}>0, \eta_{i}>0$, $i=1,2, \ldots, n$. We have

$$
\begin{aligned}
\|(1-v) A X+v X B\|_{2}^{2} \\
=\sum_{i, j=1}^{n}\left((1-v) \lambda_{i}+v \eta_{j}\right)^{2}\left|y_{i j}\right|^{2} \\
\geq \sum_{i, j=1}^{n}\left\{\left(1+\frac{L(8 v)}{16} \min \left(\log t_{i j}\right)^{2}\right)\left(\lambda_{i}^{1-v} \eta_{j}^{v}\right)^{2}+v^{2}\left(\lambda_{i}-\eta_{j}\right)^{2}+2 v\left(\lambda_{i}-\sqrt{\lambda_{i} \eta_{j}}\right)^{2}\right. \\
\left.\quad+r\left(\lambda_{i}-\sqrt[4]{\lambda_{i}^{3} \eta_{j}}\right)^{2}\right\}\left|y_{i j}\right|^{2} \quad(\mathrm{by}(2.6)) \\
=\left(1+\frac{L(8 v)}{16} \min \left(\log t_{i j}\right)^{2}\right) \sum_{i, j=1}^{n}\left(\lambda_{i}^{1-v} \eta_{j}^{v}\right)^{2}\left|y_{i j}\right|^{2}+v^{2} \sum_{i, j=1}^{n}\left(\lambda_{i}-\eta_{j}\right)^{2}\left|y_{i j}\right|^{2} \\
\quad+2 v \sum_{i, j=1}^{n}\left(\lambda_{i}-\sqrt{\lambda_{i} \eta_{j}}\right)^{2}\left|y_{i j}\right|^{2}+r \sum_{i, j=1}^{n}\left(\lambda_{i}-\sqrt[4]{\lambda_{i}^{3} \eta_{j}}\right)^{2}\left|y_{i j}\right|^{2},
\end{aligned}
$$

where $t_{i j}=\frac{\lambda_{i}}{\eta_{j}}$.

Due to the conditions $0<m \leq A \leq m^{\prime}<M^{\prime} \leq B \leq M, \frac{m}{M} \leq t_{i j}=\frac{\lambda_{i}}{\eta_{j}} \leq \frac{m^{\prime}}{M^{\prime}}=\frac{1}{h}$ and the monotonicity of the function $f(x)=\log x(0<x \leq 1)$, we get

$$
\begin{aligned}
&\|(1-v) A X+v X B\|_{2}^{2} \\
& \geq\left(1+\frac{L(8 v)}{16}(\log h)^{2}\right) \sum_{i, j=1}^{n}\left(\lambda_{i}^{1-v} \eta_{j}^{v}\right)^{2}\left|y_{i j}\right|^{2}+v^{2} \sum_{i, j=1}^{n}\left(\lambda_{i}-\eta_{j}\right)^{2}\left|y_{i j}\right|^{2} \\
&+2 v \sum_{i, j=1}^{n}\left(\lambda_{i}-\sqrt{\lambda_{i} \eta_{j}}\right)^{2}\left|y_{i j}\right|^{2}+r \sum_{i, j=1}^{n}\left(\lambda_{i}-\sqrt[4]{\lambda_{i}^{3} \eta_{j}}\right)^{2}\left|y_{i j}\right|^{2} \\
&=\left(1+\frac{L(8 v)}{16}(\log h)^{2}\right)\left\|A^{1-v} X B^{v}\right\|_{2}^{2}+v^{2}\|A X-X B\|_{2}^{2}+2 v\left\|A X-A^{\frac{1}{2}} X B^{\frac{1}{2}}\right\|_{2}^{2} \\
&+r\left\|A X-A^{\frac{3}{4}} X B^{\frac{1}{4}}\right\|_{2}^{2},
\end{aligned}
$$

where we completed the proof of (2.10). Using the same method, we can get (2.11)-(2.13) by (2.7)-(2.9), respectively, so we omit the details.

Next, we give further improvements of (1.12) and (1.13) for positive linear maps. But first, let us present the following lemmas that will be useful later. 
Lemma 2.5 ([1]) Let $\Phi$ be a unital positive linear map, $A, B$ be positive operators, and $v \in[0,1]$. Then

$$
\Phi\left(A \sharp_{\nu} B\right) \leq \Phi(A) \sharp_{\nu} \Phi(B) .
$$

Lemma 2.6 ([2]) Let $A, B \geq 0$ and $\alpha>0$. Then

$$
\left\|A^{\frac{1}{2}} B^{-\frac{1}{2}}\right\| \leq \alpha^{\frac{1}{2}} \quad \text { iff } \quad A \leq \alpha B .
$$

Lemma 2.7 ([4]) Let $A, B \geq 0$. Then the following norm inequality holds:

$$
\|A B\| \leq \frac{1}{4}\|A+B\|^{2} .
$$

Lemma 2.8 (L-H inequality, [8]) If $0 \leq p \leq 1$ and $A \geq B \geq 0$, then

$$
A^{p} \geq B^{p} .
$$

Lemma 2.9 ([1]) Let $A, B \geq 0$. Then for $1 \leq p<+\infty$,

$$
\left\|A^{p}+B^{p}\right\| \leq\left\|(A+B)^{p}\right\| .
$$

Lemma 2.10 (Choi inequality, [3]) Let $\Phi$ be a unital positive linear map and $A>0$. Then

$$
\Phi(A)^{-1} \leq \Phi\left(A^{-1}\right) .
$$

Theorem 2.11 Let $0<m I \leq A, B \leq M I, \alpha=\max \left\{\frac{(M+m)^{2}}{4 M m}, \frac{(M+m)^{2}}{4^{\frac{2}{p}} M m}\right\}, p \geq 0$, and $0 \leq v \leq \frac{1}{4}$. For every positive unital linear map $\Phi$, we have

$$
\Phi^{p}\left(A \nabla_{\nu} B+M m J\left(A^{-1} ; B^{-1}\right)\right) \leq \alpha^{p} \Phi^{p}\left(A \sharp_{\nu} B\right)
$$

and

$$
\Phi^{p}\left(A \nabla_{\nu} B+M m J\left(A^{-1} ; B^{-1}\right)\right) \leq \alpha^{p}\left(\Phi(A) \sharp_{\nu} \Phi(B)\right)^{p},
$$

where $J\left(A^{-1} ; B^{-1}\right)=2 v\left(A^{-1} \nabla B^{-1}-A^{-1} \sharp B^{-1}\right)+2 v\left(A^{-1} \sharp B^{-1}+A^{-1}-2\left(A^{-1} \sharp_{\frac{1}{4}} B^{-1}\right)\right)+$ $r\left(A^{-1} \sharp_{\frac{1}{4}} B^{-1}+A^{-1}-2\left(A^{-1} \sharp_{\frac{1}{8}} B^{-1}\right)\right)+G^{*} A^{-1} \sharp_{v} B^{-1} G$ for $G=\frac{\sqrt{L(8 v)}}{8} A S\left(A^{-1} \mid B^{-1}\right)$.

Proof For $0<m I \leq A, B \leq M I$, we have

$$
(M-A)(A-m) A^{-1} \geq 0 \quad \text { and } \quad(M-B)(B-m) B^{-1} \geq 0,
$$

that is,

$$
A+M m A^{-1} \leq M+m \quad \text { and } \quad B+M m B^{-1} \leq M+m .
$$


For every positive unital linear map $\Phi$, we have

$$
\Phi(A)+M m \Phi\left(A^{-1}\right) \leq M+m \quad \text { and } \quad \Phi(B)+M m \Phi\left(B^{-1}\right) \leq M+m
$$

So we have

$$
\begin{aligned}
& \Phi((1-v) A)+M m \Phi\left((1-v) A^{-1}\right) \leq(1-v)(M+m) \quad \text { and } \\
& \Phi(v B)+M m \Phi\left(v B^{-1}\right) \leq v(M+m)
\end{aligned}
$$

Summing up the inequalities above, we can get

$$
\Phi\left(A \nabla_{\nu} B\right)+M m \Phi\left(A^{-1} \nabla_{v} B^{-1}\right) \leq M+m .
$$

When $0 \leq v \leq \frac{1}{4}$, taking $a=1$ in (2.2), we have

$$
\begin{aligned}
& b^{v}+v\left(1+b-2 b^{\frac{1}{2}}\right)+2 v\left(1+b^{\frac{1}{2}}-2 b^{\frac{1}{4}}\right)+r\left(1+b^{\frac{1}{4}}-2 b^{\frac{1}{8}}\right) \\
& \quad+\left(\frac{\sqrt{L(8 v)}}{8} \log b\right) \times b^{v} \times\left(\frac{\sqrt{L(8 v)}}{8} \log b\right) \leq(1-v)+v b .
\end{aligned}
$$

Now by the functional calculus for the positive operator $A^{\frac{1}{2}} B^{-1} A^{\frac{1}{2}}$, we have

$$
\begin{aligned}
& \left(A^{\frac{1}{2}} B^{-1} A^{\frac{1}{2}}\right)^{v}+v\left(I+\left(A^{\frac{1}{2}} B^{-1} A^{\frac{1}{2}}\right)-2\left(A^{\frac{1}{2}} B^{-1} A^{\frac{1}{2}}\right)^{\frac{1}{2}}\right) \\
& \quad+2 v\left(I+\left(A^{\frac{1}{2}} B^{-1} A^{\frac{1}{2}}\right)^{\frac{1}{2}}-2\left(A^{\frac{1}{2}} B^{-1} A^{\frac{1}{2}}\right)^{\frac{1}{4}}\right)+r\left(I+\left(A^{\frac{1}{2}} B^{-1} A^{\frac{1}{2}}\right)^{\frac{1}{4}}-2\left(A^{\frac{1}{2}} B^{-1} A^{\frac{1}{2}}\right)^{\frac{1}{8}}\right) \\
& \quad+\left(\frac{\sqrt{L(8 v)}}{8} \log \left(A^{\frac{1}{2}} B^{-1} A^{\frac{1}{2}}\right)\right) \times\left(A^{\frac{1}{2}} B^{-1} A^{\frac{1}{2}}\right)^{v} \times\left(\frac{\sqrt{L(8 v)}}{8} \log \left(A^{\frac{1}{2}} B^{-1} A^{\frac{1}{2}}\right)\right) \\
& \leq(1-v) I+v\left(A^{\frac{1}{2}} B^{-1} A^{\frac{1}{2}}\right) .
\end{aligned}
$$

Multiplying by $A^{-\frac{1}{2}}$ both sides of inequality (2.23), we have

$$
\begin{aligned}
& A^{-1} \sharp_{\nu} B^{-1}+2 v\left(A^{-1} \nabla B^{-1}-A^{-1} \sharp B^{-1}\right)+2 v\left(A^{-1} \sharp B^{-1}+A^{-1}-2\left(A^{-1} \sharp_{\frac{1}{4}} B^{-1}\right)\right) \\
& \quad+r\left(A^{-1} \sharp_{\frac{1}{4}} B^{-1}+A^{-1}-2\left(A^{-1} \sharp_{\frac{1}{8}} B^{-1}\right)\right)+G^{*} A^{-1} \sharp_{V} B^{-1} G \\
& \leq A^{-1} \nabla_{\nu} B^{-1} .
\end{aligned}
$$

Moreover,

$$
\begin{aligned}
A^{-1} \sharp_{\nu} B^{-1}+J\left(A^{-1} ; B^{-1}\right) \\
=A^{-1} \sharp_{\nu} B^{-1}+2 v\left(A^{-1} \nabla B^{-1}-A^{-1} \sharp B^{-1}\right)+2 v\left(A^{-1} \sharp B^{-1}+A^{-1}-2\left(A^{-1} \sharp_{\frac{1}{4}} B^{-1}\right)\right) \\
\quad+r\left(A^{-1} \sharp_{\frac{1}{4}} B^{-1}+A^{-1}-2\left(A^{-{ }_{\sharp}}{ }_{\frac{1}{8}} B^{-1}\right)\right)+G^{*} A^{-1} \sharp_{V} B^{-1} G \\
\leq A^{-1} \nabla_{v} B^{-1} .
\end{aligned}
$$


So we have

$$
\begin{aligned}
\| \Phi & \left(A \nabla_{v} B+M m J\left(A^{-1} ; B^{-1}\right)\right)+M m \Phi^{-1}\left(A \sharp_{v} B\right) \| \\
& \leq\left\|\Phi\left(A \nabla_{v} B+M m J\left(A^{-1} ; B^{-1}\right)\right)+M m \Phi\left(A^{-1} \sharp_{v} B^{-1}\right)\right\| \\
& =\left\|\Phi\left(A \nabla_{v} B\right)+M m \Phi\left(J\left(A^{-1} ; B^{-1}\right)+A^{-1} \sharp_{\nu} B^{-1}\right)\right\| \\
& \leq\left\|\Phi\left(A \nabla_{v} B\right)+M m \Phi\left(A^{-1} \nabla_{v} B^{-1}\right)\right\| \\
& \leq M+m,
\end{aligned}
$$

where the first inequality is by (2.19), the second is by (2.24), and the third is by (2.22).

Therefore,

$$
\begin{aligned}
\| \Phi & \left(A \nabla_{v} B+M m J\left(A^{-1} ; B^{-1}\right)\right) M m \Phi^{-1}\left(A \sharp_{v} B\right) \| \\
& \leq \frac{1}{4}\left\|\Phi\left(A \nabla_{v} B+M m J\left(A^{-1} ; B^{-1}\right)\right)+M m \Phi^{-1}\left(A \sharp_{v} B\right)\right\|^{2} \\
& \leq \frac{(M+m)^{2}}{4},
\end{aligned}
$$

where the first inequality is by (2.16) and the second is by (2.25). That is,

$$
\left\|\Phi\left(A \nabla_{\nu} B+M m J\left(A^{-1} ; B^{-1}\right)\right) \Phi^{-1}\left(A \sharp_{v} B\right)\right\| \leq \frac{(M+m)^{2}}{4 M m} .
$$

By Lemma 2.6, we have

$$
\Phi^{2}\left(A \nabla_{\nu} B+M m J\left(A^{-1} ; B^{-1}\right)\right) \leq\left(\frac{(M+m)^{2}}{4 M m}\right)^{2} \Phi^{2}\left(A \sharp_{v} B\right) .
$$

When $0 \leq p \leq 2$, then $0 \leq \frac{p}{2} \leq 1$, hence by (2.17), we have

$$
\Phi^{p}\left(A \nabla_{\nu} B+M m J\left(A^{-1} ; B^{-1}\right)\right) \leq\left(\frac{(M+m)^{2}}{4 M m}\right)^{p} \Phi^{p}\left(A \sharp_{\nu} B\right)=\alpha^{p} \Phi^{p}\left(A \sharp_{\nu} B\right) .
$$

When $p>2$,

$$
\begin{aligned}
M^{\frac{P}{2}} & m^{\frac{P}{2}}\left\|\Phi^{\frac{p}{2}}\left(A \nabla_{v} B+M m J\left(A^{-1} ; B^{-1}\right)\right) \Phi^{-\frac{p}{2}}\left(A \sharp_{v} B\right)\right\| \\
& =\left\|\Phi^{\frac{p}{2}}\left(A \nabla_{v} B+M m J\left(A^{-1} ; B^{-1}\right)\right) M^{\frac{P}{2}} m^{\frac{P}{2}} \Phi^{-\frac{p}{2}}\left(A \sharp_{v} B\right)\right\| \\
& \leq \frac{1}{4}\left\|\Phi^{\frac{p}{2}}\left(A \nabla_{v} B+M m J\left(A^{-1} ; B^{-1}\right)\right)+M^{\frac{p}{2}} m^{\frac{p}{2}} \Phi^{-\frac{p}{2}}\left(A \sharp_{v} B\right)\right\|^{2} \\
& \leq \frac{1}{4}\left\|\left(\Phi\left(A \nabla_{v} B+M m J\left(A^{-1} ; B^{-1}\right)\right)+M m \Phi^{-1}\left(A \sharp_{v} B\right)\right)^{\frac{p}{2}}\right\|^{2} \\
& =\frac{1}{4}\left\|\Phi\left(A \nabla_{v} B+M m J\left(A^{-1} ; B^{-1}\right)\right)+M m \Phi^{-1}\left(A \sharp_{v} B\right)\right\|^{p} \\
& \leq \frac{1}{4}(M+m)^{p},
\end{aligned}
$$


where the first inequality is by (2.16), the second is by (2.18), and the third is by (2.25). That is,

$$
\left\|\Phi^{\frac{p}{2}}\left(A \nabla_{v} B+M m J\left(A^{-1} ; B^{-1}\right)\right) \Phi^{-\frac{p}{2}}\left(A \sharp_{v} B\right)\right\| \leq \frac{(M+m)^{p}}{4 M^{\frac{p}{2}} m^{\frac{p}{2}}}=\alpha^{\frac{p}{2}},
$$

which is equivalent to (2.20) by (2.15). Here we completed the proof of (2.20). We now prove (2.21) for $0 \leq p \leq 2$. Indeed,

$$
\begin{aligned}
\| \Phi & \left(A \nabla_{\nu} B+\operatorname{MmJ}\left(A^{-1} ; B^{-1}\right)\right) M m\left(\Phi(A) \sharp_{\nu} \Phi(B)\right)^{-1} \| \\
& \leq \frac{1}{4}\left\|\Phi\left(A \nabla_{\nu} B+\operatorname{MmJ}\left(A^{-1} ; B^{-1}\right)\right)+\operatorname{Mm}\left(\Phi(A) \sharp_{\nu} \Phi(B)\right)^{-1}\right\|^{2} \\
& \leq \frac{1}{4}\left\|\Phi\left(A \nabla_{v} B+\operatorname{MmJ}\left(A^{-1} ; B^{-1}\right)\right)+M m \Phi^{-1}\left(A \sharp_{\nu} B\right)\right\|^{2} \\
& \leq \frac{(M+m)^{2}}{4}
\end{aligned}
$$

where the first inequality is by (2.16), the second is by (2.14), and the third is by (2.25). That is,

$$
\left\|\Phi\left(A \nabla_{v} B+M m J\left(A^{-1} ; B^{-1}\right)\right)(\Phi(A) \sharp \Phi(B))^{-1}\right\| \leq \frac{(M+m)^{2}}{4 M m}=\alpha,
$$

so we can get (2.21) by (2.15) and (2.17) easily when $0 \leq p \leq 2$. When $p>2$,

$$
\begin{aligned}
M^{\frac{P}{2}} & m^{\frac{P}{2}}\left\|\Phi^{\frac{p}{2}}\left(A \nabla_{\nu} B+\operatorname{MmJ}\left(A^{-1} ; B^{-1}\right)\right)\left(\Phi(A) \sharp_{\nu} \Phi(B)\right)^{-\frac{p}{2}}\right\| \\
& =\left\|\Phi^{\frac{p}{2}}\left(A \nabla_{\nu} B+\operatorname{MmJ}\left(A^{-1} ; B^{-1}\right)\right) M^{\frac{P}{2}} m^{\frac{p}{2}}\left(\Phi(A) \sharp_{\nu} \Phi(B)\right)^{-\frac{p}{2}}\right\| \\
& \leq \frac{1}{4}\left\|\Phi^{\frac{p}{2}}\left(A \nabla_{\nu} B+\operatorname{MmJ}\left(A^{-1} ; B^{-1}\right)\right)+M^{\frac{P}{2}} m^{\frac{p}{2}}\left(\Phi(A) \sharp_{\nu} \Phi(B)\right)^{-\frac{p}{2}}\right\|^{2} \\
& \leq \frac{1}{4}\left\|\left(\Phi\left(A \nabla_{\nu} B+\operatorname{MmJ}\left(A^{-1} ; B^{-1}\right)\right)+M m\left(\Phi(A) \sharp_{\nu} \Phi(B)\right)^{-1}\right)^{\frac{p}{2}}\right\|^{2} \\
& =\frac{1}{4}\left\|\Phi\left(A \nabla_{\nu} B+\operatorname{MmJ}\left(A^{-1} ; B^{-1}\right)\right)+\operatorname{Mm}\left(\Phi(A) \sharp_{\nu} \Phi(B)\right)^{-1}\right\|^{p} \\
& \leq \frac{1}{4}\left\|\Phi\left(A \nabla_{\nu} B+\operatorname{MmJ}\left(A^{-1} ; B^{-1}\right)\right)+M m \Phi^{-1}\left(A \sharp_{\nu} B\right)\right\|^{p} \\
& \leq \frac{1}{4}(M+m)^{p},
\end{aligned}
$$

where the first inequality is by (2.16), the second is by (2.18), the third is by (2.14), and the last inequality is by (2.25). That is,

$$
\left\|\Phi^{\frac{p}{2}}\left(A \nabla_{\nu} B+M m J\left(A^{-1} ; B^{-1}\right)\right)\left(\Phi(A) \sharp_{\nu} \Phi(B)\right)^{-\frac{p}{2}}\right\| \leq \frac{(M+m)^{p}}{4 M^{\frac{p}{2}} m^{\frac{p}{2}}}=\alpha^{\frac{p}{2}},
$$

and we can get (2.21) by (2.15) easily. Hence we completed the proof of Theorem 2.11.

Remark 2.12 Let $0<m I \leq A, B \leq M I, v \in[0,1]$ and $r=\min \{v, 1-v\}$. It is clear that $2 v\left(A^{-1} \sharp B^{-1}+A^{-1}-2\left(A^{-1} \sharp_{\frac{1}{4}} B^{-1}\right)\right) \geq 0$ and $r\left(A^{-1} \sharp_{\frac{1}{4}} B^{-1}+A^{-1}-2\left(A^{-1} \sharp_{\frac{1}{8}} B^{-1}\right)\right) \geq 0$. In other 
words, our results can be regarded as further refinements of reversed AM-GM operator inequalities of [12].

By the same methods of Theorem 2.11, we can get further improvements of (1.12) and (1.13) for $\frac{1}{4} \leq v \leq 1$.

Corollary 2.13 Under the same conditions as in Theorem 2.11, we have:

(1) When $\frac{1}{4} \leq v \leq \frac{1}{2}$,

$$
\Phi^{p}\left(A \nabla_{\nu} B+M m F_{1}\left(A^{-1} ; B^{-1}\right)\right) \leq \alpha^{p} \Phi^{p}\left(A \sharp_{\nu} B\right)
$$

and

$$
\Phi^{p}\left(A \nabla_{\nu} B+M m F_{1}\left(A^{-1} ; B^{-1}\right)\right) \leq \alpha^{p}\left(\Phi(A) \sharp_{\nu} \Phi(B)\right)^{p},
$$

where $F_{1}\left(A^{-1} ; B^{-1}\right)=2 v\left(A^{-1} \nabla B^{-1}-A^{-1} \sharp B^{-1}\right)+(1-2 v)\left(A^{-1} \sharp B^{-1}+A^{-1}-\right.$

$\left.2\left(A^{-1} \sharp_{\frac{1}{4}} B^{-1}\right)\right)+r\left(A^{-1} \sharp_{\frac{1}{4}} B^{-1}+A^{-1} \sharp B^{-1}-2\left(A^{-1} \sharp_{\frac{3}{8}} B^{-1}\right)\right)+G^{*} A^{-1} \sharp_{v} B^{-1} G$;

(2) When $\frac{1}{2} \leq v \leq \frac{3}{4}$,

$$
\Phi^{p}\left(A \nabla_{v} B+M m F_{2}\left(A^{-1} ; B^{-1}\right)\right) \leq \alpha^{p} \Phi^{p}\left(A \sharp_{\nu} B\right)
$$

and

$$
\Phi^{p}\left(A \nabla_{\nu} B+M m F_{2}\left(A^{-1} ; B^{-1}\right)\right) \leq \alpha^{p}\left(\Phi(A) \sharp_{\nu} \Phi(B)\right)^{p},
$$

where $F_{2}\left(A^{-1} ; B^{-1}\right)=2(1-v)\left(A^{-1} \nabla B^{-1}-A^{-1} \sharp B^{-1}\right)+(2 v-1)\left(A^{-1} \sharp B^{-1}+B^{-1}-\right.$ $\left.2\left(A^{-1} \sharp_{\frac{3}{4}} B^{-1}\right)\right)+r\left(A^{-1} \sharp_{\frac{3}{4}} B^{-1}+A^{-1} \sharp B^{-1}-2\left(A^{-1} \sharp_{5} B^{-1}\right)\right)+G^{*} A^{-1} \sharp_{v} B^{-1} G$;

(3) When $\frac{3}{4} \leq v \leq 1$,

$$
\Phi^{p}\left(A \nabla_{v} B+M m F_{3}\left(A^{-1} ; B^{-1}\right)\right) \leq \alpha^{p} \Phi^{p}\left(A \sharp_{v} B\right)
$$

and

$$
\Phi^{p}\left(A \nabla_{v} B+M m F_{3}\left(A^{-1} ; B^{-1}\right)\right) \leq \alpha^{p}\left(\Phi(A) \sharp_{\nu} \Phi(B)\right)^{p},
$$

where $F_{3}\left(A^{-1} ; B^{-1}\right)=2(1-v)\left(A^{-1} \nabla B^{-1}-A^{-1} \sharp B^{-1}\right)+2(1-v)\left(A^{-1} \sharp B^{-1}+B^{-1}-\right.$ $\left.2\left(A^{-1} \sharp_{\frac{3}{4}} B^{-1}\right)\right)+r\left(A^{-1} \sharp_{\frac{3}{4}} B^{-1}+B^{-1}-2\left(A^{-1} \sharp_{\frac{7}{8}} B^{-1}\right)\right)+G^{*} A^{-1} \sharp_{v} B^{-1} G$.

In the end of this paper, we give some inequalities for determinants which were not mentioned in Yang's paper and its references. But first, we state a lemma.

Lemma 2.14 (Minkowski inequality, [6]) Let $a=\left[a_{i}\right], b=\left[b_{i}\right], i=1,2, \ldots, n$ be such that $a_{i}, b_{i}$ are positive real numbers. Then

$$
\left(\prod_{i=1}^{n} a_{i}\right)^{\frac{1}{n}}+\left(\prod_{i=1}^{n} b_{i}\right)^{\frac{1}{n}} \leq\left(\prod_{i=1}^{n}\left(a_{i}+b_{i}\right)\right)^{\frac{1}{n}} .
$$

The equality holds if and only if $a=b$. 
Theorem 2.15 Let $A, B \in M_{n}$ be two positive semidefinite matrices and $T=A^{-\frac{1}{2}} B A^{-\frac{1}{2}}$, $v \in[0,1], \widetilde{F}(v)=\min \left(1+\frac{L(8 v)}{16}\left(\log s_{j}(T)\right)^{2}\right)$. Then the following statements are true:

(1) When $0 \leq v \leq \frac{1}{4}$ and $r=\min \{4 v, 1-4 v\}$, we have

$$
\begin{aligned}
\operatorname{det}\left(A \nabla_{v} B\right)^{\frac{2}{n}} \geq & \widetilde{F}(v) \operatorname{det}\left(A \sharp_{v} B\right)^{\frac{2}{n}}+v^{2} \operatorname{det}(A-B)^{\frac{2}{n}} \\
& +2 v \operatorname{det}(A-A \sharp B)^{\frac{2}{n}}+r \operatorname{det}\left(A-A \sharp_{\frac{1}{4}} B\right)^{\frac{2}{n}} .
\end{aligned}
$$

(2) When $\frac{1}{4} \leq v \leq \frac{1}{2}$ and $r=\min \{2-4 v, 4 v-1\}$, we have

$$
\begin{aligned}
\operatorname{det}\left(A \nabla_{v} B\right)^{\frac{2}{n}} \geq & \widetilde{F}(v) \operatorname{det}\left(A \sharp_{v} B\right)^{\frac{2}{n}}+v^{2} \operatorname{det}(A-B)^{\frac{2}{n}} \\
& +(1-2 v) \operatorname{det}(A-A \sharp B)^{\frac{2}{n}}+r \operatorname{det}\left(A \sharp B-A \sharp_{\frac{1}{4}} B\right)^{\frac{2}{n}} .
\end{aligned}
$$

(3) When $\frac{1}{2} \leq v \leq \frac{3}{4}$ and $r=\min \{3-4 v, 4 v-2\}$, we have

$$
\begin{aligned}
\operatorname{det}\left(A \nabla_{v} B\right)^{\frac{2}{n}} \geq & \widetilde{F}(v) \operatorname{det}\left(A \sharp_{v} B\right)^{\frac{2}{n}}+(1-v)^{2} \operatorname{det}(A-B)^{\frac{2}{n}} \\
& +(2 v-1) \operatorname{det}(B-A \sharp B)^{\frac{2}{n}}+r \operatorname{det}\left(A \sharp B-A \sharp_{\frac{3}{4}} B\right)^{\frac{2}{n}} .
\end{aligned}
$$

(4) When $\frac{3}{4} \leq v \leq 1$ and $r=\min \{4-4 v, 4 v-3\}$, we have

$$
\begin{aligned}
\operatorname{det}\left(A \nabla_{\nu} B\right)^{\frac{2}{n}} \geq & \widetilde{F}(v) \operatorname{det}\left(A \sharp_{v} B\right)^{\frac{2}{n}}+(1-v)^{2} \operatorname{det}(A-B)^{\frac{2}{n}} \\
& +(2-2 v) \operatorname{det}(B-A \sharp B)^{\frac{2}{n}}+r \operatorname{det}\left(B-A \sharp_{\frac{3}{4}} B\right)^{\frac{2}{n}} .
\end{aligned}
$$

Proof Using inequality (2.6) and denoting the positive definite matrix as $T=A^{-\frac{1}{2}} B A^{-\frac{1}{2}}$, we have

$$
\begin{aligned}
& \widetilde{F}(v)\left(s_{j}^{v}(T)\right)^{2}+v^{2}\left(1-s_{j}(T)\right)^{2}+2 v\left(1-s_{j}^{\frac{1}{2}}(T)\right)^{2}+r\left(1-s_{j}^{\frac{1}{4}}(T)\right)^{2} \\
& \quad \leq\left((1-v)+v s_{j}(T)\right)^{2}
\end{aligned}
$$

for $j=1,2, \ldots, n$. It is a fact that the determinant of a positive definite matrix is a product of its singular values. So we have

$$
\begin{aligned}
\operatorname{det} & \left(I \nabla_{v} T\right)^{\frac{2}{n}} \\
& =\operatorname{det}\left[((1-v) I+v T)^{2}\right]^{\frac{1}{n}} \\
& =\left[\prod_{j=1}^{n}\left((1-v) I+v s_{j}(T)\right)^{2}\right]^{\frac{1}{n}} \\
& \geq\left[\prod_{j=1}^{n}\left\{\widetilde{F}(v)\left(s_{j}^{v}(T)\right)^{2}+v^{2}\left(1-s_{j}(T)\right)^{2}+2 v\left(1-s_{j}^{\frac{1}{2}}(T)\right)^{2}+r\left(1-s_{j}^{\frac{1}{4}}(T)\right)^{2}\right\}\right]^{\frac{1}{n}} \\
& \geq\left[\prod_{j=1}^{n}\left\{\widetilde{F}(v)\left(s_{j}^{v}(T)\right)^{2}\right\}\right]^{\frac{1}{n}}+\left[\prod_{j=1}^{n}\left\{v^{2}\left(1-s_{j}(T)\right)^{2}\right\}\right]^{\frac{1}{n}}+\left[\prod_{j=1}^{n}\left\{2 v\left(1-s_{j}^{\frac{1}{2}}(T)\right)^{2}\right\}\right]^{\frac{1}{n}}
\end{aligned}
$$




$$
\begin{aligned}
&+\left[\prod_{j=1}^{n}\left\{r\left(1-s_{j}^{\frac{1}{4}}(T)\right)^{2}\right\}\right]^{\frac{1}{n}} \\
&=\widetilde{F}(v) \operatorname{det}\left(T^{v}\right)^{\frac{2}{n}}+v^{2} \operatorname{det}(I-T)^{\frac{2}{n}}+2 v \operatorname{det}\left(I-T^{\frac{1}{2}}\right)^{\frac{2}{n}}+r \operatorname{det}\left(I-T^{\frac{1}{4}}\right)^{\frac{2}{n}} .
\end{aligned}
$$

The first inequality is obtained by (2.36), while the second by Lemma 2.14. Multiplying by $\left(\operatorname{det} A^{\frac{1}{2}}\right)^{\frac{2}{n}}$ both sides of inequalities above, we can get the desired inequality (2.32) directly. Using the same technique above in (2.7)-(2.9), we can (2.33)-(2.35), respectively. To keep our paper concise, we omit the details.

\section{Acknowledgements}

Our deepest gratitude goes to the anonymous reviewers for their careful work and thoughtful suggestions.

\section{Funding}

This research is supported by the National Natural Science Foundation of China (11671201).

\section{Availability of data and materials}

The datasets used during the current study are available from the corresponding author on reasonable request.

\section{Competing interests}

The authors declare that they have no competing interests.

\section{Authors' contributions}

All author contributed to each part of this work equally, and they all read and approved the final manuscript.

\section{Publisher's Note}

Springer Nature remains neutral with regard to jurisdictional claims in published maps and institutional affiliations.

Received: 15 November 2019 Accepted: 20 March 2020 Published online: 01 April 2020

\section{References}

1. Ando, T., Zhan, X.: Norm inequalities related to operator monotone functions. Math. Ann. 315, 771-780 (1999)

2. Bakherad, M.: Refinements of a reversed AM-GM operator inequality. Linear Multilinear Algebra 64, 1687-1695 (2016)

3. Bhatia, R.: Positive Definite Matrices. Princeton University Press, Princeton (2007)

4. Bhatia, R., Kittaneh, F.: Notes on matrix arithmetic-geometric mean inequalities. Linear Algebra Appl. 308, 203-211 (2000)

5. Fu, X.H., He, C.J.: Some operator inequalities for positive linear maps. Linear Multilinear Algebra 63, 571-577 (2015)

6. Horn, R.A., Johnson, C.R.: Matrix Analysis, 2nd edn. Cambridge University Press, New York (2013)

7. Kórus, P.: A refinement of Young's inequality. Acta Math. Hung. 153, 430-435 (2017)

8. Kubo, F., Ando, T.: Means of positive linear operators. Math. Ann. 246, 205-224 (1980)

9. Liao, W.S., Wu, J.L.: Improved Kantorovich and Wielandt operator inequalities for positive linear maps. Filomat 31 , $871-876(2017)$

10. Lin, M.: Squaring a reverse AM-GM inequality. Stud. Math. 215, 187-194 (2013)

11. Lin, M.: On an operator Kantorovich inequality for positive linear maps. J. Math. Anal. Appl. 402, 127-132 (2013)

12. Yang, C.J., Gao, Y.X., Lu, F.Y.: Some refinements of young type inequality for positive linear map. Math. Slovaca 69, 919-930 (2019)

13. Yang, C.J., Lu, F.Y.: Improving some operator inequalities for positive linear maps. Filomat 32, 4333-4340 (2018)

14. Yang, C.S., Wang, D.H.: Some refinements of operator inequalities for positive linear maps. J. Math. Inequal. 11, 17-26 (2017)

15. Yang, C.S., Yang, C.J.: Further generalizations of some operator inequalities involving positive linear map. Filomat 31, 2355-2364 (2017)

16. Zou, L.M.: An arithmetic-geometric mean inequality for singular values and its applications. Linear Algebra Appl. 528, 25-32 (2017)

17. Zou, L.M., Jiang, Y.Y.: Improved arithmetic-geometric mean inequality and its application. J. Math. Inequal. 9, 107-111 (2015)

18. Zou, L.M., Jiang, Y.Y.: A note on interpolation between the arithmetic-geometric mean and Cauchy-Schwarz matrix norm inequalities. J. Math. Inequal. 10, 1119-1122 (2016) 